EPJ Web of Conferences 62, 07001 (2013)

DOI: $10.1051 /$ epjconf/20136207001

(C) Owned by the authors, published by EDP Sciences, 2013

\title{
Influence of orientation degree of freedom on fission dynamics of higly excited nuclei
}

\author{
P.N. Nadtochy ${ }^{a}$, E.G. Ryabov, A.E. Gegechkori, Yu.A. Anischenko, and G.D. Adeev \\ Omsk State University, Mira prospekt 55-A, Omsk 644077, Russia
}

\begin{abstract}
Four-dimensional dynamical model was developed and employed for study fission characteristics in a wide range of fissility parameter. The three collective shape coordinates plus the $\mathrm{K}$ coordinate, which is the spin of the nucleus with respect to the symmetry (fission) axis, were considered dynamically from the ground state deformation till the scission into fission fragments. A modified one-body mechanism for nuclear dissipation with a reduction coefficient $k_{s}$ of the contribution from a "wall" formula have been used in the study. The four-dimensional calculations for heavy nuclei could describe the fission fragment mass-energy distribution (MED) parameters and prescission neutron multiplicity with almost single $k_{s}$ value, in contrast with 3D dynamical calculations, where a consistent description of all observables with the same $k_{s}$ is not possible for heavy nuclei. The estimation of a dissipation coefficient for the orientation degree of freedom $\gamma_{K}=0.077$ $(\mathrm{MeV} \mathrm{zs})^{-1 / 2}$ is good for heavy nuclei and lower value of $\gamma_{K}=0.05(\mathrm{MeV} \mathrm{zs})^{-1 / 2}$ is needed for nuclei with mass $\mathrm{A} \simeq 200$. The results of $4 \mathrm{D}$ and 3D Langevin dynamical calculations for light nuclei near the Businaro-Gallone point predict close results for the fission fragment MED parameters and prescission particles multiplicities.
\end{abstract}

\section{Introduction}

The nuclear fission is a magnificent phenomenon of a quantal large-scale collective motion during which the substantial rearrangement of initial compound nucleus results in its splitting on two or more parts. However, the character of the compound nucleus evolution is still not well established, and it is not yet understood in detail how the original compound nucleus is transformed into a variety of fission products. During last decades stochastic approach based on multidimensional Langevin equations has been extensively and rather successfully used to elucidate many problems of collective nuclear dynamics in fusion-fission reactions at high excitation energies [1,2]. It was shown in our previous studies, that in order to describe the mass-energy distribution (MED) of fission fragments the three independent shape parameters are needed [3] and optimal choice of the collective degrees of freedom allow to describe a wide range of experimental data [4, 5]. Numerous three-dimensional (3D) Langevin calculations [2] have shown the capability of the model to reproduce experimental data for compound nuclei of different fissility obtained in fusion-fission reactions.

\footnotetext{
ae-mail: p.nadtochy@gsi.de
}

This is an Open Access article distributed under the terms of the Creative Commons Attribution License 2.0, which permits unrestricted use, distribution, and reproduction in any medium, provided the original work is properly cited. 
Recently the significance of orientation degree of freedom ( $\mathrm{K}$ coordinate), which is the projection of the total angular momentum $(I)$ onto the symmetry axis of fissioning nucleus, was demonstrated for the studies based on statistical model $[6,7]$. In these papers an overdamped Langevin equation for $\mathrm{K}$ coordinate were introduced. At present many models do not consider the evolution of $\mathrm{K}$ coordinate, and assume the $\mathrm{K}$ fixed and equal to zero. The transition state model at saddle (or scission) point is implemented in order to calculate the angular distribution of fission fragments. In this case two inconsistencies are admitted. First, skipping the influence of non-zero K values on the potential energy surface during dynamical evolution. Second, the assumption that equilibration of $\mathrm{K}$ coordinate will be reached at the saddle or scission point. Therefore, recently the three-dimensional dynamical model was elaborated to take into account the fourth collective coordinate (K-coordinate) [5]. This coordinate could substantially influence the dynamical evolution of fissioning system and predicted parameters of fission fragments mass-energy distribution as well as on the fission timescale [5, 7]. Inclusion of this coordinate into three-dimensional dynamical model allowed for the first time to have unified dynamical description of mass-energy distribution together with angular distribution of fission fragments.

The first results of the dynamical calculations based on three-dimensional Langevin model plus K coordinate (4D) [5] demonstrated the advantage of such calculations, since the 4D dynamical model allows consistent calculations of fission fragment MED, prescission particle multiplicities and the fission fragments angular distribution. The calculations performed in Ref. [5] have shown that the 4D model could describe the fission fragment MED parameters and prescission particles multiplicities for heavy nuclei using almost the same dissipation strength, which is not possible within 3D calculations. In the present study we investigate in detail the influence of the K-coordinate on the fission rate, time scale, and fission observables in a wide interval of fissility parameter. The results of the present study could be very useful for the qualitative estimations of the influence of K coordinate in the models, which skip the consideration of this coordinate.

\section{Model}

In the present study we used a stochastic approach to treat fission process at high excitation energy $[1,2,4,5,8]$. We used recently developed 4D dynamical model for the description of fissioning nucleus shape evolution. The detailed description of the model could be found in Refs. [2, 5] and here we only give a short description of basic ingredients. In the present study a modified one-body mechanism of nuclear dissipation [9-12] was employed to determine the dissipative part of the driving forces with reduction coefficient from the "wall" formula $k_{s}$. The value $k_{s}=1.0$ corresponds to the "wall" and "wall-plus-window" formulas, whereas values $0.2<k_{s}<0.5$ allow to reproduce different features of the experimental fission fragment MED and particle multiplicities in multidimensional Langevin calculations [2, 13-15]. The values $k_{s}<1$ are also compatible with several theoretical predictions $[11,16-18]$.

The description of evolution of the $K$ collective coordinate using the Langevin equation for overdamped regime has been recently proposed in Ref. [7]:

$$
\delta K=-\frac{\gamma_{K}^{2} I^{2}}{2} \frac{\partial V}{\partial K} \delta t+\gamma_{K} I \xi \sqrt{T \delta t},
$$

where $\xi$ is a random number from a normal distribution with unit variance. The $\gamma_{K}$ is a friction parameter controlling the coupling between the orientation degree of freedom $K$ and the "heat bath".

The Langevin equations for the shape parameters and Langevin equation for the $K$ coordinate (1) are connected through the potential energy. The Langevin dynamics of $K$ coordinate is influenced by the actual value of potential energy $V(\mathbf{q}, I, K)$. At the same time, the rotational part of the potential energy depends on the $K$ value at time $t$. 

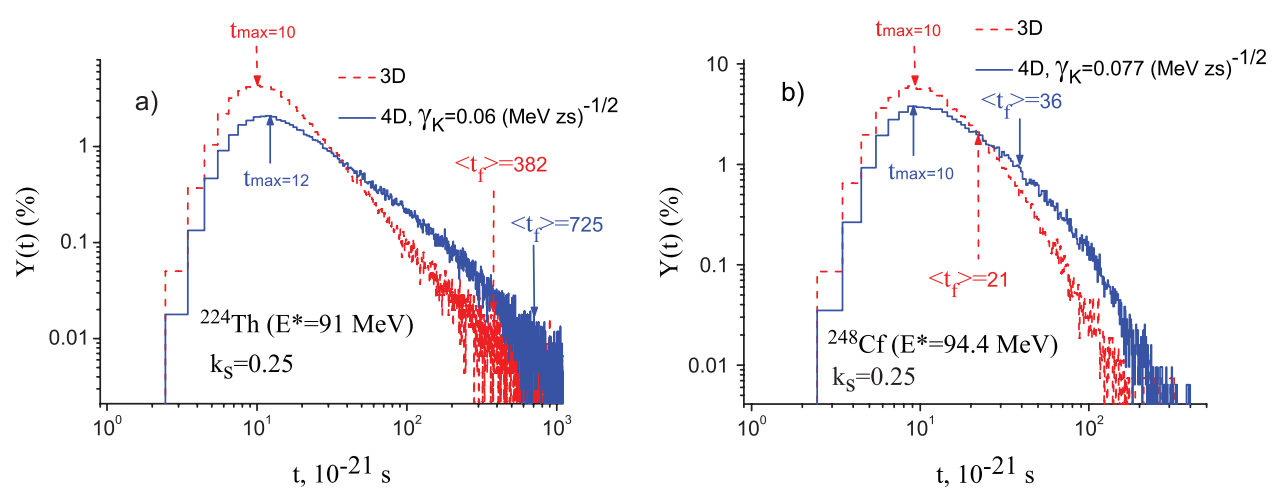

Figure 1. The fission time distribution for the ${ }^{224} \mathrm{Th}$ (a) and ${ }^{248} \mathrm{Cf}$ (b) fissioning nuclei in $3 \mathrm{D}$ (dashed histogram) and $4 \mathrm{D}$ (solid histogram) calculations obtained with $k_{s}=0.25$ and different $\gamma_{K}$ values.

We choose $\gamma_{K}$ to be a constant equal to $0.077(\mathrm{MeV} \mathrm{zs})^{-1 / 2}$ following to the estimations made in Ref. [7]. This estimation has been obtained for elongated nuclear shapes featuring a neck, which corresponds to the deformations typical for the descent from saddle to scission point. Since this estimate may be incorrect by a factor of 2 or more [7], we also performed calculations with different $\gamma_{K}$ values, trying to explore the sensitivity of observables to the $k_{S}$ and $\gamma_{K}$ parameters. In our previous calculations we have found that $\gamma_{K} \simeq 0.077(\mathrm{MeV} \mathrm{zs})^{-1 / 2}$ is appropriate for the description of the anisotropy of angular distribution for the highly excited fissioning compound nuclei with mass $A_{C N} \simeq 230-250$.

\section{Results and discussions}

In the present study we investigate in detail the influence of $\mathrm{K}$ coordinate on time distribution of fission events. We perform 3D and 4D calculations for the ${ }^{224} \mathrm{Th}$ and ${ }^{248} \mathrm{Cf}$ compound nuclei with $k_{s}=0.25$ and different $\gamma_{K}$ values, which reproduce the anisotropy of fission fragment angular distribution for these nuclei [5] . The resulting time distributions are presented in Figure 1.

A fission delay time $\tau_{d}=3 \mathrm{zs}$ is found for both nuclei in 3D and 4D calculations. The $\tau_{d}{ }^{\text {' determines }}$ the time interval $0<t<\tau_{d}$ where no fission events occur. In the time interval $\tau_{d}<t<t_{f}^{\max }$ the fission time distribution has steep rise from 0 to maximum value. At $t>t_{f}^{\max }$ the fission time distribution is approximately exponential decreasing function with a long lasting tail up to $10^{-18} \mathrm{~s}$. The $t_{f}^{\max }$ value is affected very little by the dimensionality of the dynamical model. On the other hand the incline of the tail is the characteristic mostly affected by the dimensionality. As a result the mean fission time $\left\langle t_{f}\right\rangle$, indicated by the arrow, demonstrates the strongest sensitivity to the inclusion of K coordinate. Different incline of time distribution tails in 3D and 4D calculations in Figure 1 is the reflection of the fission rate decrease in 4D calculations in comparison with 3D ones. In our previous study [5] we have found that this decrease is caused by the appearance of additional conservative force slowing down the motion in the fission direction and the increase of fission barrier height after inclusion of non-zero $K$ values in the dynamical consideration.

The influence of $\mathrm{K}$ coordinate on $\left\langle n_{\text {pre }}\right\rangle$ and $\sigma_{M}^{2}$ is presented in Figure 2 for the ${ }^{224} \mathrm{Th}$ compound nucleus. In this figure the results of 3D calculations [4] are compared with the results of 4D calculations with the similar value of $k_{s}$ coefficient. It was noticed in Ref. [4] that in order to describe the variance of mass distribution $\sigma_{M}^{2}$ one needs to use the small values $k_{s} \simeq 0.25$, but in order to describe the prescission neutron multiplicities one needs to use large $k_{s}$ values around 1 . The similar results were obtained in other study [21]. Thus, the 3D model does not allow to describe the experimental data on $\left\langle n_{\text {pre }}\right\rangle$ and $\sigma_{M}^{2}$ with a single $k_{s}$ value for heavy nuclei $[2,3]$. After the inclusion of $\mathrm{K}$ coordinate into dynamical 

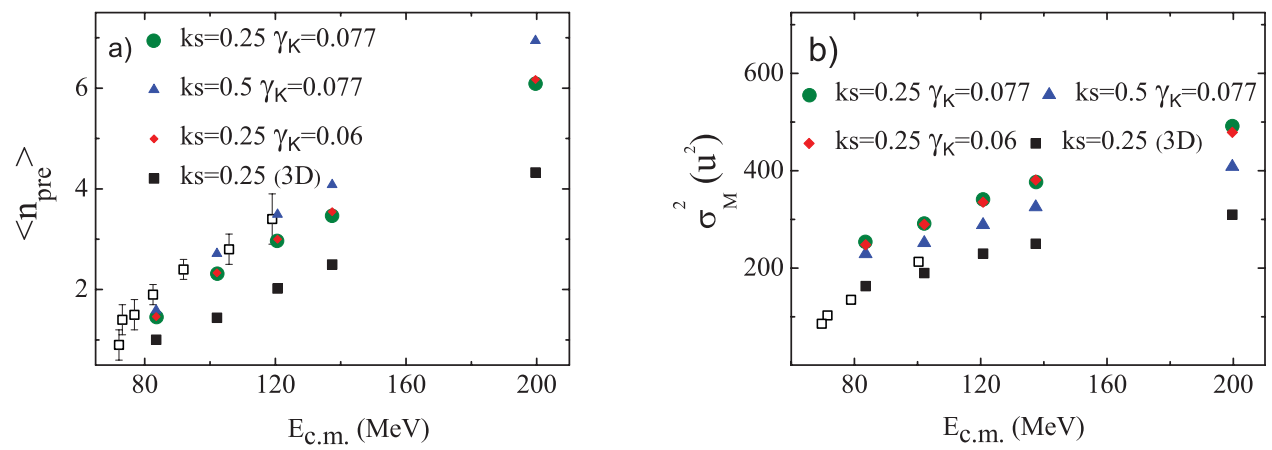

Figure 2. The calculated prescission neutron multiplicity (a) and variance of fission fragment mass distribution (b) are compared with experimental data $[19,20]$ for the ${ }^{224} \mathrm{Th}$. The open symbols are experimental data, the filled circles are results of 4D calculations with $k_{s}=0.25$ and $\gamma_{K}=0.077(\mathrm{MeV} \mathrm{zs})^{-1 / 2}$, the filled triangles - results of 4D calculations with $k_{s}=0.5$ and $\gamma_{K}=0.077(\mathrm{MeV} \mathrm{zs})^{-1 / 2}$, the filled dimonds - results of 4D calculations with $k_{s}=0.25$ and $\gamma_{K}=0.06(\mathrm{MeV} \mathrm{zs})^{-1 / 2}$, and the filled squares $-3 \mathrm{D}$ calculations with $k_{s}=0.25$.
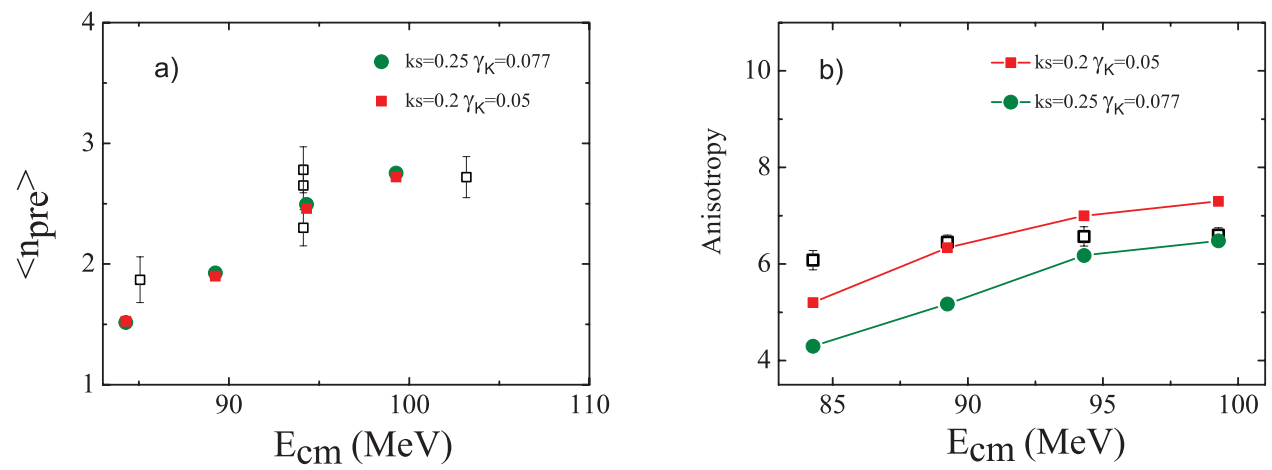

Figure 3. The prescission neutron multiplicity (a) and anisotropy of angular distribution (b) obtained for the ${ }^{200} \mathrm{~Pb}$. The open symbols are experimental data [22, 23], the filled circles are results of 4D calculations with $k_{s}=0.25$ and $\gamma_{K}=0.077(\mathrm{MeV} \mathrm{zs})^{-1 / 2}$, the filled squares $-4 \mathrm{D}$ calculations with $k_{s}=0.2$ and $\gamma_{K}=0.05(\mathrm{MeV} \mathrm{zs})^{-1 / 2}$.

modeling both characteristics could be described with almost single $k_{s}$ value. Such result is guaranteed by the increase of fission barrier height and decrease of the potential energy stiffness with respect to mass-asymmetry coordinate [5]. In this sense the K coordinate helps to resolve the puzzling task [3, 4] of describing the $\left\langle n_{\text {pre }}\right\rangle$ and $\sigma_{M}^{2}$ for the region of heavy nuclei.

In the present study we investigate the influence of $\mathrm{K}$ coordinate on the fission dynamics for nucleus ${ }^{200} \mathrm{~Pb}$ formed in the reaction ${ }^{16} \mathrm{O}+{ }^{184} \mathrm{~W} \rightarrow{ }^{200} \mathrm{~Pb}\left(E_{\text {lab }}=91.6,97.7,102.5\right.$, and $\left.107.9 \mathrm{MeV}\right)$. The results of $4 \mathrm{D}$ calculations in comparison with experimental data are presented in Figure 3 for the $\left\langle n_{\text {pre }}\right\rangle$ and anisotropy of angular distribution. This figure shows that good reproduction of $\left\langle n_{\text {pre }}\right\rangle$ could be obtained using one-body dissipation mechanism with reduction factor $k_{s}=0.2-0.25$ independently of $\gamma_{K}$ and anisotropy of angular distribution could be reproduced with $k_{s}=0.2$ and $\gamma_{K}=0.5(\mathrm{MeV} \mathrm{zs})^{-1 / 2}$.

In order to investigate influence of the $\mathrm{K}$ coordinate for light nuclei we performed $4 \mathrm{D}$ calculations for three fissioning systems: ${ }^{105} \mathrm{Ag}\left(E^{*}=121 \mathrm{MeV}\right),{ }^{132} \mathrm{Ce}\left(E^{*}=122 \mathrm{MeV}\right)$, and ${ }^{172} \mathrm{Yb}\left(E^{*}=128 \mathrm{MeV}\right)$. As there is no experimental data on angular distribution for these nuclei and $\gamma_{K}$ does not influence on the parameters of fission fragment MED and prescission particles multiplicities [5], there is no possibility to estimate the $\gamma_{K}$, which will fit experimental anisotropy of fission fragment angular distribution. Therefore, for simplicity we use the $\gamma_{K}=0.077(\mathrm{MeV} \mathrm{zs})^{-1 / 2}$, the same as for the heavy nuclei [5]. 
Fission 2013
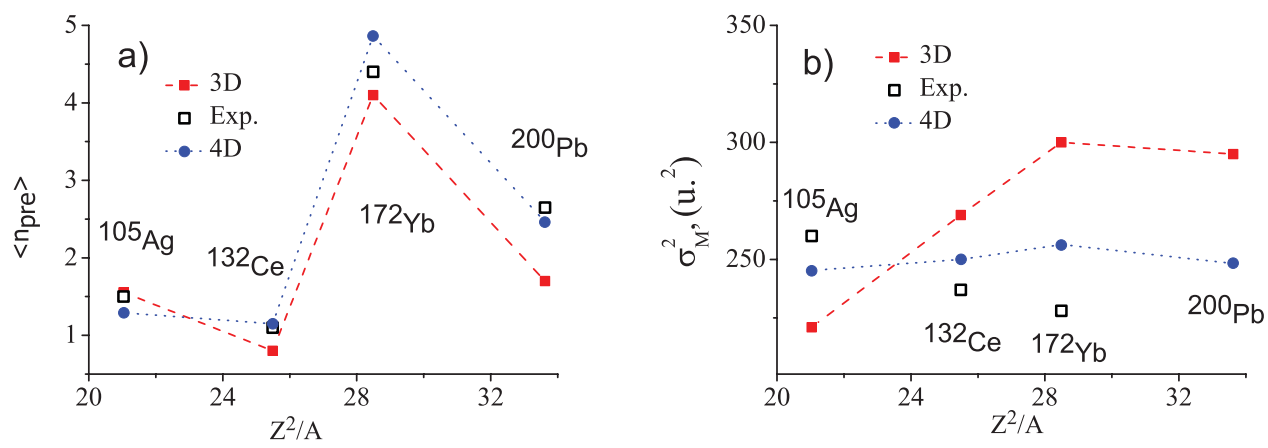

Figure 4. The prescission neutron multiplicity (a) and variance of fission fragment mass distribution (b) obtained for the different nuclei. The open symbols are experimental data, the filled circles are results of 4D calculations with different $k_{s}$ values (see text for details) and $\gamma_{K}=0.077(\mathrm{MeV} \mathrm{zs})^{-1 / 2}$, the filled squares are results of corresponding $3 \mathrm{D}$ calculations.

We tune $k_{s}$ value in order to fit available experimental data on $\left\langle n_{\text {pre }}\right\rangle$ and $\sigma_{M}^{2}$ for these nuclei in 4D calculations. As a results we found that $k_{s}=0.5,1.0$, and 0.25 are needed in order to describe $\left\langle n_{\text {pre }}\right\rangle$ and $\sigma_{M}^{2}$ for the ${ }^{105} \mathrm{Ag},{ }^{132} \mathrm{Ce}$, and ${ }^{172} \mathrm{Yb}$, respectively. As a next step we performed the 3D calculations for these nuclei with the same $k_{s}$ values as in 4D calculations. Thus, we can estimate how large will be the influence of the K coordinate on calculated observables. The results of the corresponding 3D and 4D calculations are presented in Figure 4.

Analysis shows that $\left\langle n_{\text {pre }}\right\rangle$ and $\sigma_{M}^{2}$ values only slightly depend on the K coordinate for the lightest considered nuclei ${ }^{105} \mathrm{Ag}$ and ${ }^{132} \mathrm{Ce}$. In case of heavier nuclei ${ }^{172} \mathrm{Yb}$ and ${ }^{200} \mathrm{~Pb}$ the difference between $3 \mathrm{D}$ and $4 \mathrm{D}$ calculations becomes larger. It is about 20 and $30 \%$ for the $\sigma_{M}^{2}$ and $\left\langle n_{\text {pre }}\right\rangle$, respectively.

\section{Conclusions}

From our present investigation we can conclude that the 4D calculations for heavy nuclei could describe the fission fragment MED parameters and prescission particles multiplicities with almost single $k_{s}$ value, which is not possible within 3D calculations. The estimation of $\gamma_{K}=0.077(\mathrm{MeV} \mathrm{zs})^{-1 / 2}$ is good for heavy nuclei and lower value of $\gamma_{K}=0.05(\mathrm{MeV} \mathrm{zs})^{-1 / 2}$ for nuclei with mass $\mathrm{A} \simeq 200$. The results of 4D and 3D calculations for light nuclei near the Businaro-Gallone point predict close results for MED parameters and prescission particles multiplicities.

The work was supported by the Russian Foundation for Basic Research, Research Project No. 13-02-00168 (Russia).

\section{References}

[1] Y. Abe, S. Ayik, P-G. Reinhard, and E. Suraud, Phys. Rep. 275, 49 (1996)

[2] G.D. Adeev et al., Fiz. Elem. Chast. At. Yadra 36, 732 (2005)

[3] A.V. Karpov, P.N. Nadtochy, D.V. Vanin, and G.D. Adeev, Phys. Rev. C 63, 054610 (2001)

[4] P.N. Nadtochy, G.D. Adeev, A.V. Karpov, Phys. Rev. C 65, 064615 (2002)

[5] P.N. Nadtochy, E.G. Ryabov, A.E. Gegechkori, Yu.A. Anischenko, and G.D. Adeev, Phys. Rev. C 85, 064619 (2012)

[6] J. P. Lestone, Phys. Rev. C 59, 1540 (1999) 
[7] J. P. Lestone and S. G. McCalla, Phys. Rev. C 79, 044611 (2009)

[8] H.A. Kramers, Physica 7, 284 (1940)

[9] J. Blocki, Y. Boneh, J.R. Nix, J. Randrup, M. Robel, A.J. Sierk, W.J. Świạtecki, Ann. Phys. (N.Y.) 113, 330 (1978)

[10] J. Randrup and W. J. Swiatecki, Nucl. Phys. A 429, 105 (1984)

[11] J.R. Nix, A.J. Sierk, in Proceedings of the International School-Seminar on Heavy Ion Physics, Dubna, USSR, 1986, ed. by M.I. Zarubina, E.V. Ivashkevich (JINR, Dubna, 1987), pp. 453-464

[12] J.R. Nix, A.J. Sierk, in Proceedings of the 6th Adriatic Conference on Nuclear Physics: Frontiers of Heavy Ion Physics, Dubrovnik, Yugoslavia, 1987, ed. by N. Cindro, R. Caplar, W. Greiner (World Sci., Singapore, 1990), pp. 333-340

[13] P.N. Nadtochy, A.V. Karpov, G.D. Adeev, Yad. Fiz. 65, 832 (2002) [Phys. At. Nucl. 65, 799 (2002)]

[14] P.N. Nadtochy et al., Yad. Fiz. 66, 1240 (2003) [Phys. At. Nucl. 66, 1203 (2003)]

[15] E.G. Ryabov et al., Phys. Rev. C 78, 044614 (2008)

[16] J.J. Griffin, M. Dworzecka, Nucl. Phys. A 455, 61 (1986)

[17] T. Mukhopadhyay, S. Pal, Phys. Rev. C 56, 296 (1997)

[18] S. Pal, T. Mukhopadhyay, Phys. Rev. C 57, 210 (1998)

[19] H. Rossner et al., Phys. Rev. C 45, 719 (1992)

[20] M. G. Itkis et al., in Proceedings of the XV EPS Conference on Low Energy Nuclear Dynamics (LEND-95), St. Peterburg, 1995, edited by Yu. Ts. Oganessian, R. Kalpakchieva, and W. von Oertzen (World Scientific, Singapore, 1995), p. 177

[21] J. Wilczyński, K. Siwek-Wilczyńska, and H. W. Wilschut, Phys. Rev. C 54, 325 (1996)

[22] J. S. Forster et al., Nucl. Phys. A 464, 497 (1987)

[23] J. O. Newton et al., Nucl. Phys. A 483, 126 (1988) 